\title{
Nucleation and growth of metal nanoparticles on a planar electrode: a new model based on iso-nucleation-time classes of particles
}

Pietro Altimari ${ }^{\mathrm{a}, *}$, Francesco Greco ${ }^{\mathrm{b}, *}$, Francesca Pagnanelli ${ }^{\mathrm{a}}$

${ }^{a}$ Dipartimento di Chimica, Università Sapienza di Roma, Piazzale Aldo Moro 5, 00185 Rome, Italy

b Dipartimento di Ingegneria Chimica, dei Materiali e della Produzione Industriale, Università degli Studi di Napoli Federico II, Piazzale Tecchio, 80125 Naples, Italy

\begin{abstract}
An assembly of hemispherical particles continuously nucleating on a planar electrode and growing under mixed kinetic-diffusion control is here considered. A model is derived, from the exact boundary integral formulation of the diffusion equation, to predict the overall current evolution, and the radii distribution of particles nucleating within any prescribed time interval. Iso-nucleation-time classes are introduced in the model, grouping particles (almost) simultaneously nucleating over the underlying substrate. The dynamics of particles belonging to a given iso-nucleation time class are assumed to be identical. By this approximation, hereby referred to as Averaged Class Approximation (ACA), the computation of the average radius of any iso-nucleation-time class is reduced to the solution of an integro-differential equation, parameterized by the nucleation time. An effective computational method is also presented to solve the model equations, giving predictions that fairly well agree with the results of direct multi-particle numerical simulations.
\end{abstract}

Keywords: Electrodeposition, nucleation, metal nanoparticles, three-dimensional growth, mixed kinetic-diffusion control, diffusion controlled growth.

\section{Introduction}

\footnotetext{
* Correspondence about this article should be addressed to Pietro Altimari at pietro.altimari@uniroma1.it and Francesco Greco at francesco.greco@unina.it.
} 
The physicochemical characteristics of metal nanoparticles largely depend on their size and morphology [1-3]. This has motivated a considerable interest towards the development of methods allowing for the morphology-controlled synthesis of metal nanoparticles [4]. In numerous applications, the immobilization of metal nanoparticles on foreign conductive substrates is required. In this case, electrodeposition becomes a competitive synthesis route [5].

Major advantages of electrodeposition, as compared to alternative techniques including, e.g., lithography and chemical vapour deposition, are reduced costs and versatility. Reduced costs are ensured by the direct deposition of nanoparticles onto the target substrate, without the recourse to any preparatory action, while versatility is determined by the large range of sizes and morphologies that can be attained by varying the electrodeposition parameters [6-8] (Siegfried and Choi, 2005; Kuo and Huang, 2010; Pagnanelli et al., 2015). Thus, understanding the mechanisms of electrodeposition can pave the way to the synthesis of supported metal nanoparticles with tailored physicochemical characteristics.

Metal electrodeposition on a foreign substrate is induced by supersaturation of the electrolyte solution, which can be obtained by varying the metal ion concentration $c_{0}$ or (more commonly) the electrode potential. It has been traditionally agreed that such deposition process includes the formation of active nuclei at sites randomly distributed over the electrode surface $[9,10]$. Under "stationary nucleation" conditions $[10]$, the temporal evolution of the surface nuclei number density $\mathrm{N}(\mathrm{t})$ is given by:

$N(t)=N_{0}\left[1-\exp \left(-t / t_{N}\right)\right]$

where $t, t_{N}$ and $N_{0}$ denote, respectively, the running time, the nucleation characteristic time, and the surface density of particles attained at $t>>t_{N}$, hereafter referred to as the saturation number density. Growth of nucleated particles then proceeds through diffusion of metal ions from the bulk electrolytic solution (with $D$ the diffusion coefficient), and by charge-transfer reaction at particle-solution interface (with $\mathrm{k}_{\mathrm{g}}$ the charge-transfer kinetic constant).

It should be immediately pointed out that the above illustrated representation of electrodeposition has been revised over the past few years [11-13]. According to these 
recent studies, in the very early stage of electrodeposition, the formation of nanoclusters occurs with a characteristic size around a few nanometres. These nanoclusters are stable, i.e., they do not grow. Rather, diffusion and aggregation of these nanoclusters over the electrode surface leads to the formation of particles which can successively grow through direct attachment of metal ions from the electrolytic solution. In order to simplify our analysis, however, we will stick to the traditional representation of electrodeposition, where these primordial stages are neglected. $\mathrm{N}_{0}$ and $\mathrm{t}_{\mathrm{N}}$ (Eq. (1)) must then be intended as giving the number density and the rate of appearance, respectively, of the growing particles only.

The entire electrodeposition process at constant electrode potential is typically characterized by a current transient with the shape qualitatively sketched in Fig.1, with a maximum at a certain time $t_{\max }$, followed by a monotonic decrease towards zero current [14]. (We will never consider here the case where the current, after the maximum, goes down to a constant value [15].) The presence of a maximum in the current can be understood in terms of boundary diffusion layers forming around the growing particles, and of the overlapping of such layers at sufficiently large times (Fig.1). For this to occur, the ratio between the characteristic times of diffusion $1 /\left(\mathrm{DN}_{0}\right)$ and of charge transfer $1 /\left(\mathrm{k}_{\mathrm{g}} \sqrt{\mathrm{N}_{0}}\right)$ needs, of course, to be much larger than or around unity (diffusion regime or mixed kinetic-diffusion regime, respectively).

Whatever the growth regime, size distribution and morphology of the electrodeposited particles will depend on the nucleation characteristic time $t_{N}$ and the saturation number density $\mathrm{N}_{0}[16]$. By way of example, a transition from dendritic to compact particles can be obtained under both diffusion and mixed kinetic-diffusion regimes by decreasing $t_{N}$ and/or increasing $N_{0}$ [16-19]. Likewise, it was evidenced that $N_{0}$ and $t_{N}$ can influence the spatial distribution of nuclei [20], which can ultimately affect the current transient [21] and the growth kinetics of individual particles [22]. Therefore, it is fundamental to characterize the dependence of $\mathrm{N}_{0}$ and $\mathrm{t}_{\mathrm{N}}$ on the electrodeposition parameters, i.e., as stated above, electrode potential and bulk metal ion concentration. To this purpose, mathematical models giving the current transient parametrically in $\mathrm{N}_{0}$ and $t_{N}$ can be fitted to chronoamperometric data $[14,15,23,24]$.

A major obstacle in the development of such mathematical models is represented by the need to describe the overlapping of the boundary diffusion layers. To this purpose, 
detailed modelling approaches can be implemented including, for example, Brownian dynamics [25,26], Monte Carlo simulations [27], and boundary integral methods [28,29]. However, these approaches are characterized by an elevated computational burden and are therefore disadvantageous to perform nonlinear fitting of chronoamperometric data. Rather, fitting of chronoamperometric data requires the implementation of models that can either be solved in closed analytical form or ensure a computationally efficient numerical solution [14].

An effective model satisfying these requirements was proposed by Scharifker and Mostany more than thirty years ago [24]. Scharifker and Mostany described the overlapping of the boundary diffusion layers around particles by the introduction of what they called "the diffusion zones". These are defined as the electrode areas towards which, by planar diffusion, the same flux would be transferred as that transferred, by three-dimensional spherical diffusion, to the growing particles, as if they were isolated. The overall flux of metal ions to the particles is then simply computed, in this model, by multiplying the planar diffusion flux times the fraction of the electrode surface covered by the diffusion zones; the latter fraction is computed by recourse to the Avrami theorem [30].

The analysis of Scharifker and Mostany (SM) was restricted to the diffusion regime, giving a compact analytical expression for the dependence of current density on $t_{N}$ and $N_{0}$ [24]. This expression has gained enormous success, and has been extensively used to extract estimates of $t_{N}$ and $N_{0}$ from chronoamperometric data [14]. Modifications of the SM model were proposed by several authors [31-33]. However, any proposed modification has maintained the main idea of the SM model, i.e., the introduction of the diffusion zones.

The main limitation of the SM model is that it is not rigorously derived from the diffusion equation governing the growth of nucleating particles. Specifically, the physical meaning of the diffusion zones introduced to describe the overlapping of the boundary diffusion layers remains unclear. This makes it difficult to implement the method of diffusion zones to cover deviations from three-dimensional diffusion-controlled growth, which might include, for example, the occurrence of mixed kinetic-diffusion regime [34,35] (Altimari and Pagnanelli, 2016a,b), or the non-negligible contribution of adatom and nanocluster diffusion to growth [12]. Furthermore, the SM model does not provide any information on the particle size distribution at any time. 
An alternative way to compute the current transient was proposed by Bobbert et al. (1987) [36]. In contrast to the SM approach, Bobbert's method relies on the rigorous, albeit approximate, analysis of the balance equations that govern diffusion to an assembly of particles (hereafter referred to as the multi-particle diffusion problem). Particles were described by point-wise sinks, and an approximate solution for the current transient was obtained by recourse to a "mean field approximation", whereby the concentration of metal ions only varies with the distance from the electrode and, accordingly, the same concentration of metal ions is invariably found at the surface of any particle. This simplification reduced the computation of the current transient to the solution of an integro-differential equation. Good agreement was found between the current transients predicted by the integro-differential equation and by the numerical solution the multiparticle diffusion problem, respectively. However, the following limitations remain in the work by Bobbert et al. (1987) [36]: i) only the case of instantaneous nucleation is treated; ii) his analysis only covers the purely diffusion regime.

In the present article, we propose a model overcoming the above described limitations. The model relies on the approximation that particles nucleating at (essentially) the same time are identical, i.e., undergo the same growth history, wherever they are born on the electrode surface. By this approximation, the analysis of the electrodeposition problem is reduced to the solution of an integro-differential equation parameterized by the nucleation time. Solving the latter equation allows to get an (approximate) particles radii distribution, from which the current transient can be obtained. In contrast to the models by Scharifker and Mostany (1984) [24] and Bobbert et al. (1987) [36], our model applies to the general case of mixed kinetic-diffusion regime.

The paper is organized as follows. In section 2.1, the problem of metal electrodeposition on a foreign substrate is recast in the form of a multi-particle diffusion problem. In section 2.2, the application of the boundary integral method to obtain the "exact solution" of the multi-particle diffusion problem is illustrated. In section 2.3, the new model is described, to predict the radii distribution of particles nucleating within any prescribed time interval and the overall current evolution. In section 2.4, a coarse-graining of the new model is presented to facilitate the numerical solution. In section 3 , the results of the model are compared with the "exact solution" of the multi-particle diffusion problem. Some final remarks are included in the conclusions section. 


\section{Computation of the current transient}

\subsection{Multi-particle diffusion problem}

A population of active sites distributed with number density $\mathrm{N}_{0}$ over a planar electrode is considered. We assume that the electrode area is infinitely large, which allows neglecting edge effects, and is immersed in a quiescent electrolyte solution with uniform initial concentration $c_{0}$ of metal ions. We in fact consider the half-space problem only, i.e., with the electrolyte solution only wetting one side of the electrode. Supersaturation is imposed at $\mathrm{t}=0$ (for example, by application of sufficiently large cathodic potential), which causes metal particles to continuously nucleate and grow at the active sites, with finite rate $1 / t_{N}$ (Eq. (1)). Particles are assumed to be hemispherical, and to grow under mixed kineticdiffusion control, with first-order irreversible charge-transfer taking place at particlesolution interface. Notice that we will neglect ion migration induced by electric potential gradients, which is a proper approximation under the condition of excess of nonelectroactive ions (of a "supporting electrolyte" [37]).

We assume that metal ions can only be discharged or deposited on the particles, implying that the diffusion flux of the metal ions at the substrate surface is zero. With this assumption, the problem of diffusion to hemispherical particles on a substrate is equivalent to the whole-space problem of diffusion to spherical particles with centres in the same plane. To verify this equivalence, let us consider the equations governing the diffusion of metal ions to an assembly of spherical particles. The concentration field $c(r, t)$ can be determined by solving the following diffusion equation:

$$
\frac{\partial c(\mathbf{r}, t)}{\partial t}=D \nabla^{2} c(\mathbf{r}, t)
$$

with initial and boundary conditions given by Eq.(3) and Eqs.(4)-(5), respectively

$$
\begin{aligned}
& \mathrm{c}=\mathrm{c}_{0} \quad \forall \mathbf{r}, \quad \text { at } \mathrm{t}=0 \\
& \mathbf{n}_{\mathrm{i}} \cdot(\mathrm{D} \nabla \mathrm{c})=\mathrm{k}_{\mathrm{g}} \mathrm{c}, \quad \mathbf{r} \in \bigcup_{\mathrm{i}} \mathrm{A}_{\mathrm{i}}, \forall \mathrm{t} \\
& \mathrm{c}=\mathrm{c}_{0}, \quad|\mathrm{z}| \rightarrow+\infty, \forall \mathrm{t}
\end{aligned}
$$


where $D$ is the diffusion coefficient, $\mathrm{k}_{\mathrm{g}}$ is the charge transfer kinetic constant (also referred to as growth kinetic constant), $A_{i}$ is the surface of the spherical particle $i, \mathbf{n}_{\mathbf{i}}$ is the outward unit normal vector to particle surface $A_{i}$, and $z$ is the distance from the plane where spherical particles centres lie. In fact, Eqs. (2)-(5) express all and the same conditions on the concentration field $c(r, t)$ that would be imposed in the problem of half-space diffusion to hemispherical particles on a substrate, but the one requesting that the diffusion flux of metal ions normal to the substrate (hence, along z) is zero. On the other hand, since the centres of the spherical particles are in the same plane, symmetry ensures that no metal ion can ever cross such plane, which allows one to solve the half-space problem with hemispherical particles through Eqs. (2)-(5).

The growth of particles implies time-varying boundaries $A_{i}(t)$. The following set of mass balance equations must therefore be coupled to Eqs. (1)-(5) to track the evolution of particle radii (and thus of the boundaries $\mathrm{A}_{\mathrm{i}}$ ):

$\frac{d}{d t}\left(\frac{4}{3} \pi R_{i}^{3} \frac{1}{v_{M}}\right)=\int_{A_{i}} D \nabla c \cdot n_{i} d A_{i}=\int_{A_{i}} k_{g} c d A_{i}, \quad R_{i}\left(t_{i}\right)=R_{0}$

where $v_{M}$ denotes the metal molar volume, and $t_{i}$ is the nucleation time of particle $i$. Notice that all particles are assumed to nucleate at active sites with the same finite radius $R_{0}$. Notice, further, that the second equality in Eq. (6) directly follows from Eq. (4) above. Once the concentration field $c(r, t)$ and the radii $R_{i}$ are determined, the electric current I through the electrode can be computed by summing up the fluxes of metal ions to the hemispherical particles, and then multiplying times the molar transferred charge, which gives:

$I=\frac{z F}{2} \sum_{i} \int_{A_{i}} k_{g} c d A_{i}$

where $\mathrm{z}$ and $\mathrm{F}$ denote the valence of the precursor metal ion and the Faraday's constant, respectively. Of course, index $i$ varies between 1 and the number of particles currently active over the electrode surface. The factor $1 / 2$ multiplying the Right Hand Side (RHS) of 
Eq. (7) is introduced to take into account that the diffusion flux to the hemispherical particles is half the diffusion flux to spherical ones.

\subsection{Boundary integral formulation}

The diffusion problem Eqs. (2)-(5) can be solved by application of the boundary integral method [38]. With this method, the concentration $c(r, t)$ at any point $\mathbf{r}$ belonging to the union of particles' surfaces can be directly obtained from:

$$
\frac{1}{2} c(\mathbf{r}, t)=c_{0}-\sum_{i} \int_{t_{i}}^{t} d t^{\prime} \int_{A_{i}^{\prime}} d A_{i}^{\prime} C\left(\mathbf{r}^{\prime}, t^{\prime}\right)\left[k_{g} G\left(\mathbf{r}, t \mid \mathbf{r}^{\prime}, t^{\prime}\right)-D \mathbf{n}_{i}^{\prime} \cdot \nabla G\left(\mathbf{r}, t \mid \mathbf{r}^{\prime}, t^{\prime}\right)\right], \quad \mathbf{r} \in \bigcup_{i} A_{i}
$$

where the $\mathrm{i}$ index runs over the set of particles existing at current time $t$, and $G\left(\mathbf{r}, t \mid \mathbf{r}^{\prime}, \mathrm{t}^{\prime}\right)$ is the three-dimensional Green's function of the diffusion equation:

$G\left(\mathbf{r}, t \mid \mathbf{r}^{\prime}, t^{\prime}\right)=\frac{\exp \left[-\frac{\left\|\mathbf{r}-\mathbf{r}^{\prime}\right\|^{2}}{4 D\left(t-t^{\prime}\right)}\right]}{\left[4 \pi D\left(t-t^{\prime}\right)\right]^{3 / 2}}$

The i-th term of the sum in Eq. (8) simply gives the contribution of particle $\mathrm{i}$ to the concentration field at position $\mathbf{r}$ and time $\mathrm{t}$. Such contribution is calculated by integrating over the surface $A_{i}^{\prime}$ (integration in $\mathbf{r}^{\prime}$ ) and over all times (integration in $t^{\prime}$ ) from the nucleation time $t_{i}$ of particle $i$ to the current time $t$.

Therefore, by adopting the boundary integral method, the equations governing the evolution of the metal ion concentration at particle surfaces and the evolution of the particle radii become Eqs. (6) and (8). It is convenient now to rewrite these equations in dimensionless form. We adopt the following choice: length and time are scaled by the average interparticle distance $\mathrm{N}_{0}^{-1 / 2}$ and the diffusion characteristic time $1 / \mathrm{DN}_{0}$, respectively, and concentration is scaled by $\mathrm{c}_{0}$. This gives:

$$
\frac{d}{d \tau}\left(\frac{4}{3} \pi Y_{i}^{3}\right)=v_{M} K_{g} \int_{S_{i}} w(y, \tau) d S_{i}, \quad Y_{i}\left(\tau_{i}\right)=Y_{0}
$$




$$
\frac{1}{2} w(\mathbf{y}, \tau)=1-\sum_{i} \int_{\tau_{i}}^{\tau} d \tau^{\prime} \int_{S_{i}^{\prime}} d S_{i}^{\prime} w\left(\mathbf{y}^{\prime}, \tau^{\prime}\right)\left[K_{g} \Gamma\left(\mathbf{y}, \tau \mid \mathbf{y}^{\prime}, \tau^{\prime}\right)-\mathbf{n}_{i}^{\prime} \cdot \nabla \Gamma\left(\mathbf{y}, \tau \mid \mathbf{y}^{\prime}, \tau^{\prime}\right)\right], \quad \mathbf{y} \in \bigcup_{i} S_{i}
$$

with $\Gamma\left(\mathbf{y}, \tau \mid \mathbf{y}^{\prime}, \tau^{\prime}\right)$ the dimensionless Green's function:

$$
\Gamma\left(\mathbf{y}, \tau \mid \mathbf{y}^{\prime}, \tau^{\prime}\right)=\frac{\exp \left[-\frac{\left\|\mathbf{y}-\mathbf{y}^{\prime}\right\|^{2}}{4\left(\tau-\tau^{\prime}\right)}\right]}{\left[4 \pi\left(\tau-\tau^{\prime}\right)\right]^{3 / 2}}
$$

and with dimensionless parameters defined by Eq.(13):

$$
\mathrm{K}_{\mathrm{g}}=\frac{\mathrm{k}_{\mathrm{g}}}{\mathrm{D} \sqrt{\mathrm{N}_{0}}} ; \quad v_{\mathrm{M}}=\frac{\mathrm{C}_{0}}{1 / \mathrm{v}_{\mathrm{M}}}
$$

Finally, the number of growing particles changes with time according to the dimensionless form of Eq. (1):

$$
\mu(\tau)=1-\exp \left(-\tau / \tau_{N}\right)
$$

where $\tau_{\mathrm{N}}=\mathrm{t}_{\mathrm{N}} /\left(1 / \mathrm{DN}_{0}\right)$ is the dimensionless nucleation characteristic time. According to the above reported equations, electrodeposition dynamics are governed by three dimensionless parameters: the ratio $\mathrm{K}_{\mathrm{g}}$ between the characteristic rate of charge transfer reaction and the diffusion rate, the ratio $\tau_{N}$ of the characteristic nucleation time to the diffusion time, and the ratio $v_{M}$ of the metal ion concentration in the solution to the metal molar density.

\subsection{The "exact solution" of the multi-particle diffusion problem}

The following two assumptions are introduced at this stage, to proceed with the computation of particle radii and particle surface concentrations: 
i. The concentration of metal ions is assumed to be uniform over the surface of any particle, which allows the introduction of particle concentrations $w_{i}(\tau)$, i.e., it is $\mathrm{w}(\mathbf{y}, \tau)=\mathrm{w}_{\mathrm{i}}(\tau)$ at any $\mathbf{y} \in \mathrm{S}_{\mathrm{i}}$;

ii. Interparticle distance is always much larger than any particle radius, that is $Y_{i}(\tau)<<1 / \sqrt{\mu(\tau)}$ (in dimensional form: $R_{i}(t)<<1 / \sqrt{N(t)}$ )

By virtue of these assumptions, Eq. (11) can be rewritten as a list of equations for the particle concentrations $\mathrm{w}_{\mathrm{i}}(\tau)$, as follows:

$$
\begin{aligned}
& \frac{1}{2} W_{i}(\tau)=1-\sum_{j, j \neq i \tau_{j}}^{\tau} \int_{\tau_{j}} d \tau^{\prime} \frac{\exp \left[-\frac{Y_{i j}^{2}}{4\left(\tau-\tau^{\prime}\right)}\right]}{2 \sqrt{\pi}\left(\tau-\tau^{\prime}\right)^{3 / 2}}\left(K_{g} Y_{j}\left(\tau^{\prime}\right)^{2}-Y_{j}\left(\tau^{\prime}\right)\right) W_{j}\left(\tau^{\prime}\right) \\
& \quad+\int_{\tau_{i}}^{\tau} d \tau^{\prime} \frac{1}{\sqrt{4 \pi\left(\tau-\tau^{\prime}\right)}}\left[K_{g}+\frac{1}{Y_{i}\left(\tau^{\prime}\right)}-\left(K_{g}+\frac{1}{Y_{i}\left(\tau^{\prime}\right)}+\frac{Y_{i}\left(\tau^{\prime}\right)}{\left(\tau-\tau^{\prime}\right)}\right) \exp \left(-\frac{Y_{i}\left(\tau^{\prime}\right)^{2}}{\left(\tau-\tau^{\prime}\right)}\right)\right] W_{i}\left(\tau^{\prime}\right)
\end{aligned}
$$

where $\mathrm{y}_{\mathrm{ij}}=\left\|\mathbf{y}_{\mathrm{i}}-\mathbf{y}_{\mathrm{j}}\right\|$, with $\mathbf{y}_{\mathrm{i}}$ and $\mathbf{y}_{\mathrm{j}}$ the positions occupied by the centres of particle $\mathrm{i}$ and $\mathrm{j}$, respectively. The derivation of Eq. (15) is presented in the Appendix. It is worth remarking that the last integral appearing on the RHS of Eq. (15) comes from the term of Eq. (11) that expresses the contribution of particle $i$ to the concentration $w_{i}(\tau)$. This term was not accounted for in the derivation presented by Bobbert et al. (1987) [36]. These latter authors approximated particles by point-wise sources, and this approximation is clearly inadequate to compute the contribution of particle $i$ to the concentration field $w_{i}(\tau)$. This introduces an error in the solution of the multi-particle diffusion problem, which is in turn transferred to the computation of the mean particle concentration. We excluded this error by discarding the point-wise approximation for particle $i$, and accounting for the effect of the finite particle radius in the computation of the concentration field $w_{i}(\tau)$.

According to the assumption of uniform particle surface concentration, Eq. (10) is also simplified:

$$
\frac{d Y_{i}}{d \tau}=v_{M} K_{g} W_{i}(\tau), \quad Y_{i}\left(\tau_{i}\right)=Y_{0}
$$


Eqs. (14)-(16) give a set of integro-differential equations, which can be numerically solved to compute the evolution of the particle concentrations $w_{i}(\tau)$ and radii $Y_{i}(\tau)$. The number of equations Eqs.(15)-(16) is two times the number of nucleated particles, which varies with time in accordance with Eq. (14). For computational purposes, only a fraction of the electrode surface is considered, which corresponds to select a finite number of active sites. Numerical solution of Eqs. (14)-(16) was derived in the present study by the method proposed by Cao and West (2002) [29]. In this method, a discretization of the time domain is introduced, and a Monte Carlo approach is implemented to simulate the nucleation of particles at active sites, so as to enforce the temporal evolution given by Eq. (14). It has to be noticed that numerical simulations performed with this method are invariably characterized by an elevated computational burden, which is mainly determined by the following reasons: an extremely small step size and an elevated number of active sites must be imposed to attain numerical convergence over a time sufficient to cover the entire evolution of electrodeposition, well beyond the overlapping of the boundary diffusion layers forming around particles (see the introduction). As numerical simulations with an increasing number of active sites (increasing electrode area) are performed, current transient will eventually approach its "true" shape.

In the present study, numerical simulations were repeated for any selected set of parameter values by increasing the number of active sites until the maximum relative variation in the computed current density was lower than $5 \%$. Convergence tests were also performed to verify that the computed numerical solution was not sensitive to variations in the adopted time step size.

\subsection{Averaged Class Approximation}

As illustrated in the previous section, electrodeposition dynamics are completely determined by the temporal evolution of two distributions, namely, the distributions of particle radii $Y_{i}$ and concentrations $w_{i}$. Such distributions are of course affected by the spatial arrangement of the active sites and by the progressive nucleation of particles: in other words, two growing particles differ from each other because of their ages and of their spatial positions. Indeed, the spatial distribution of the active sites may introduce differences between the surroundings of particles, e.g., differences in the distances from their first neighbours. A shorter distance from first neighbours can, for example, decrease 
the concentration of metal ion at particle surface (competition in the uptake of metal ions from the solution is increased), and can thus decrease the particle growth rate. Likewise, if the difference between the nucleation times of particles $i$ and $j$ is much larger than the characteristic time of particle growth, particles $i$ and $j$ will invariably exhibit different radii, i.e., $Y_{i} \neq Y_{j}$.

In order to simplify the analysis, we assume hereafter that particles nucleating at (essentially) the same time are, irrespective of the sites where they nucleate, identical, in the sense that they will always exhibit identical radii and surface concentrations of the metal ion. In view of this assumption, it proves convenient to introduce the iso-nucleationtime class of particles $C_{\mathrm{u}}$ as the set of particles with nucleation time belonging to the time interval $[u, u+d u]$. The entire set of nucleated particles up to the current time $\tau$ is then obtained by the union of the iso-nucleation-time classes $C_{u}$, with $u$ varying between 0 and $\tau$. The assumption that particles $i$ and $j$ nucleating at (essentially) the same time are identical means that $w_{i}(\tau)=w_{j}(\tau)$ and $Y_{i}(\tau)=Y_{j}(\tau)$. The metal ion concentration attained at particles belonging to a prescribed iso-nucleation-time class $C_{u}$ can thus be expressed through a unique concentration $\mathrm{w}(\tau, \mathrm{u})$, depending on $\tau$ and $\mathrm{u}$. In the same way, a unique radius $\mathrm{Y}(\tau, \mathrm{u})$ can be defined for particles belonging to $C_{\mathrm{u}}$. In words, we will refer to this description as the Averaged Class Approximation (ACA). Within such description, $\mathrm{w}(\tau, \mathrm{u})$ and $Y(\tau, u)$ will be termed as the continuous class concentration and the continuous class radius, respectively, where "continuous" refers to the fact that the birth time $u$ is now a continuous variable, $\mathrm{u} \in[0, \tau]$.

By replacing $w_{i}(\tau)$ with $w(\tau, u)$ in Eq. $(16)$, we obtain:

$$
\frac{\partial \mathrm{Y}(\tau, \mathrm{u})}{\partial \tau}=\mathrm{K}_{\mathrm{g}} v_{\mathrm{M}} \mathrm{W}(\tau, \mathrm{u}), \quad \mathrm{Y}(\mathrm{u}, \mathrm{u})=\mathrm{Y}_{0}
$$

To obtain the analogous of Eq. (15) in the ACA representation, we have in general to keep into account the spatial arrangement of the active sites (which, in Eq. (15), is described by the terms $y_{i j}$ ). In the simplest case, which is the one considered here, active sites are homogeneously distributed over the electrode surface at any time, and the evolution of the concentration $w(\tau, u)$ is given by: 


$$
\begin{aligned}
& \frac{1}{2} w(\tau, u)=1-\int_{0}^{\tau} d u^{\prime} \frac{d \mu\left(u^{\prime}\right)}{d u^{\prime}} \int_{0}^{+\infty} d \lambda 2 \pi \lambda \int_{u^{\prime}}^{\tau} d \tau^{\prime} \frac{\exp \left[-\frac{\lambda^{2}}{4\left(\tau-\tau^{\prime}\right)}\right]}{2 \sqrt{\pi}\left(\tau-\tau^{\prime}\right)^{3 / 2}}\left(K_{g} Y^{2}\left(\tau^{\prime}, u^{\prime}\right)-Y\left(\tau^{\prime}, u^{\prime}\right)\right) w\left(\tau^{\prime}, u^{\prime}\right)+ \\
& \quad+\int_{u}^{\tau} d \tau^{\prime} \frac{1}{\sqrt{4 \pi\left(\tau-\tau^{\prime}\right)}}\left[K_{g}+\frac{1}{Y\left(\tau^{\prime}, u\right)}-\left(K_{g}+\frac{1}{Y\left(\tau^{\prime}, u\right)}+\frac{Y\left(\tau^{\prime}, u\right)}{\left(\tau-\tau^{\prime}\right)}\right) \exp \left(-\frac{Y^{2}\left(\tau^{\prime}, u\right)}{\left(\tau-\tau^{\prime}\right)}\right)\right] w\left(\tau^{\prime}, u\right)
\end{aligned}
$$

The last term on the RHS of Eq. (18) is a "self-contribution term", giving the contribution of the singled-out class $C_{\mathrm{u}}$ to the concentration $\mathrm{w}(\tau, \mathrm{u})$ as a time integral between $\mathrm{u}$ and the current time $\tau$. The second term on the RHS of Eq. (18) gives the sum of the contributions of all the classes $C_{u^{\prime}}$, with $u^{\prime} \neq u$. This latter term includes a spatial integration over the electrode surface, where the surface integral is in polar coordinates (with $\lambda$ the radial coordinate) and, in view of the assumption of a homogeneous spatial arrangement of the active sites, the isotropic angular contribution is $2 \pi$. The complete contribution of the $C_{u^{\prime}}$ classes is then obtained through a double time integration, the innermost one being from the birth time of class $C_{\mathrm{u}^{\prime}}$ to the current time $\tau$, the outermost one being from 0 to $\tau$, weighted by the $\mu$ function. Indeed, $d \mu\left(u^{\prime}\right)$ is the fraction of the active sites that "switches on" over the interval $\left[u^{\prime}, u^{\prime}+d u^{\prime}\right]$.

Solving the surface integral over the interval $\lambda \in[0,+\infty[$, and substituting Eq. (14) into Eq. (18), we eventually obtain:

$$
\begin{aligned}
& \frac{1}{2} W(\tau, u)=1-\int_{0}^{\tau} d u^{\prime} \frac{\exp \left(-u^{\prime} / \tau_{N}\right)}{\tau_{N}} \int_{u^{\prime}}^{\tau} d \tau^{\prime} \sqrt{\frac{4 \pi}{\left(\tau-\tau^{\prime}\right)}}\left(K_{g} Y^{2}\left(\tau^{\prime}, u^{\prime}\right)-Y\left(\tau^{\prime}, u^{\prime}\right)\right) w\left(\tau^{\prime}, u^{\prime}\right)+ \\
& \quad+\int_{u}^{\tau} d \tau^{\prime} \frac{1}{\sqrt{4 \pi\left(\tau-\tau^{\prime}\right)}}\left[K_{g}+\frac{1}{Y\left(\tau^{\prime}, u\right)}-\left(K_{g}+\frac{1}{Y\left(\tau^{\prime}, u\right)}+\frac{Y\left(\tau^{\prime}, u\right)}{\left(\tau-\tau^{\prime}\right)}\right) \exp \left(-\frac{Y^{2}\left(\tau^{\prime}, u\right)}{\left(\tau-\tau^{\prime}\right)}\right)\right] w\left(\tau^{\prime}, u\right)
\end{aligned}
$$


Eq. (19) is an integro-differential equation parameterized by the birth time $u$ of class $C_{\mathrm{u}}$ and is coupled to Eq. (17) to determine $w(\tau, u)$ and $Y(\tau, u)$. Once these functions are known, the dimensionless current transient $J_{v}=\mathrm{IN}_{0}^{1 / 2} /\left(\mathrm{zFDC}_{0}\right)$ is determined as follows:

$$
J_{v}(\tau)=K_{g} \int_{0}^{\tau} \frac{\exp \left(-u^{\prime} / \tau_{N}\right)}{\tau_{N}} W\left(\tau, u^{\prime}\right) 2 \pi Y\left(\tau, u^{\prime}\right)^{2} d u^{\prime}=\int_{0}^{\tau} \frac{\exp \left(-u^{\prime} / \tau_{N}\right)}{\tau_{N}} \frac{2 \pi}{3 v_{M}}\left(\frac{d}{d \tau} Y\left(\tau, u^{\prime}\right)^{3}\right) d u^{\prime}
$$

The ACA model proposed here is therefore fully defined by Eqs. (17) and (19).

Notice that, in the case of instantaneous nucleation, i.e., when all particles nucleate at the same time $\tau=0$, the solution of the ACA model reduces to the determination of $\mathrm{W}(\tau, 0)=\mathrm{W}(\tau)$ and $\mathrm{Y}(\tau, 0)=\mathrm{Y}(\tau)$.

\subsection{Coarse-graining of the ACA model}

Eqs. (17) and (19) are parameterized in terms of the continuous nucleation time $u$, hence their solution is a continuous set of scalar functions of the current time $\tau$, namely, $w(\tau, u)$ and $Y(\tau, u)$. In order to proceed to a numerical solution of these equations, we adopt a coarse-grained discretization of the nucleation time domain, as follows.

By arbitrarily fixing a "coarse" time span $\delta$, we subdivide the total time interval $[0, \tau]$ into the set of intervals $\left\{\mathrm{I}_{\mathrm{i}}=\left[\mathrm{u}_{\mathrm{i}}, \mathrm{u}_{i+1}\right]\right\}_{i=1 \ldots N}$, with $\mathrm{u}_{\mathrm{i}}=(\mathrm{i}-1) \delta$, plus the remainder interval $\Omega_{N+1}=\left[u_{N+1}, \tau\right]$. Notice that, in general, if $u^{\prime}$ and $u^{\prime \prime}$ are two distinct nucleation times both falling in the same $I_{i}$, it is $w\left(\tau^{\prime}, u^{\prime}\right) \neq w\left(\tau^{\prime}, u^{\prime \prime}\right)$ for any $\tau^{\prime}$. We will instead assume that $w\left(\tau^{\prime}, u^{\prime}\right)=w\left(\tau^{\prime}, u^{\prime \prime}\right)=w\left(\tau^{\prime}, u_{i}\right)$. In other words, we will assume that differences between the concentrations of classes nucleating over a sufficiently coarsened time span $\delta\left(\mathrm{I}_{\mathrm{i}}\right.$ in the present description) can in fact be neglected. In the same way, we will assume that $w\left(\tau^{\prime}, u^{\prime}\right)=w\left(\tau^{\prime}, u^{\prime \prime}\right)=w\left(\tau^{\prime}, u_{N+1}\right)$ if $u^{\prime}$ and $u^{\prime \prime}$ are two distinct nucleation times both falling in the remainder interval $\Omega_{\mathrm{N}+1}=\left[\mathrm{u}_{\mathrm{N}+1}, \tau\right]$. With this representation, the continuous class concentration $w(\tau, u)$ is uniquely determined by the finite set of concentrations $\left\{w\left(\tau, u_{i}\right)\right\}_{i=1 \ldots N+1}$.

As a consequence of our approximation on concentrations, Eq.(17) can be integrated to give: 


$$
\begin{aligned}
Y\left(\tau, u^{\prime}\right)= & Y_{0}+\int_{u^{\prime}}^{\tau} K_{g} v_{M} w\left(\tau^{\prime}, u^{\prime}\right) d \tau^{\prime}= \\
& Y_{0}+\int_{u_{i}}^{\tau} K_{g} v_{M} W\left(\tau^{\prime}, u_{i}\right) d \tau^{\prime}-\int_{u_{i}}^{u^{\prime}} K_{g} v_{M} W\left(\tau^{\prime}, u_{i}\right) d \tau^{\prime}= \\
& Y_{0}+Y\left(\tau, u_{i}\right)-Y\left(u^{\prime}, u_{i}\right)
\end{aligned}
$$

where $u_{i}$ is the largest discretized time such that $u_{i}<u^{\prime}$. The continuous class radius $Y(\tau, u)$ is thus uniquely determined by the finite set of radii $\left\{Y\left(\tau, u_{i}\right)\right\}_{i=1 \ldots N+1}$.

In conclusion, the solution of Eqs. (17) and (19) is reduced to the determination of the $2 N+2$ functions $\left\{w\left(\tau, u_{i}\right), Y\left(\tau, u_{i}\right)\right\}_{i=1 \ldots N+1}$. It should be noticed that the number of the unknown functions grows with $\tau$, since $N+1$ is given by the integer division of $\tau$ to the fixed time span $\delta$.

The equations for the radii are simply, from Eq. (17):

$$
\frac{d Y\left(\tau, u_{i}\right)}{d \tau}=K_{g} v_{M} w\left(\tau, u_{i}\right), \quad Y\left(u_{i}, u_{i}\right)=Y_{0}
$$

The $N+1$ equations for the concentrations $w\left(\tau, u_{i}\right)$ follow from Eq. (19):

$$
\begin{aligned}
& \frac{1}{2} w\left(\tau, u_{i}\right)= \\
& 1-\sum_{k=1}^{N} \int_{u_{k}}^{u_{k+1}} d u^{\prime} \frac{\exp \left(-u^{\prime} / \tau_{N}\right)}{\tau_{N}} \int_{u^{\prime}}^{\tau} d \tau^{\prime} \sqrt{\frac{4 \pi}{\left(\tau-\tau^{\prime}\right)}}\left(K_{g} Y^{2}\left(\tau^{\prime}, u^{\prime}\right)-Y\left(\tau^{\prime}, u^{\prime}\right)\right) w\left(\tau^{\prime}, u_{k}\right) \\
& -\int_{u_{N+1}}^{\tau} d u^{\prime} \frac{\exp \left(-u^{\prime} / \tau_{N}\right)}{\tau_{N}} \int_{u^{\prime}}^{\tau} d \tau^{\prime} \sqrt{\frac{4 \pi}{\left(\tau-\tau^{\prime}\right)}}\left(K_{g} Y^{2}\left(\tau^{\prime}, u^{\prime}\right)-Y\left(\tau^{\prime}, u^{\prime}\right)\right) w\left(\tau^{\prime}, u_{N+1}\right) \\
& +\int_{u_{i}}^{\tau} d \tau^{\prime} \frac{1}{\sqrt{4 \pi\left(\tau-\tau^{\prime}\right)}}\left[K_{g}+\frac{1}{Y\left(\tau^{\prime}, u_{i}\right)}-\left(K_{g}+\frac{1}{Y\left(\tau^{\prime}, u_{i}\right)}+\frac{Y\left(\tau^{\prime}, u_{i}\right)}{\left(\tau-\tau^{\prime}\right)}\right) \exp \left(-\frac{Y^{2}\left(\tau^{\prime}, u_{i}\right)}{\left(\tau-\tau^{\prime}\right)}\right)\right] w\left(\tau^{\prime}, u_{i}\right)
\end{aligned}
$$


where the continuous class radii $Y\left(\tau^{\prime}, u^{\prime}\right)$ are obtained from Eq. $(21)$, and the $Y\left(\tau^{\prime}, u_{i}\right)$ comes directly from Eq. (22). Eqs. (22)-(23) are a set of $2 \mathrm{~N}+2$ integro-differential equations in the $2 \mathrm{~N}+2$ unknowns $\left\{\mathrm{w}\left(\tau, \mathrm{u}_{\mathrm{i}}\right), \mathrm{Y}\left(\tau, \mathrm{u}_{\mathrm{i}}\right)\right\}_{\mathrm{i}=1 \ldots \mathrm{N}+1}$.

Once $\left\{\mathrm{w}\left(\tau, \mathrm{u}_{\mathrm{i}}\right), \mathrm{Y}\left(\tau, \mathrm{u}_{\mathrm{i}}\right)\right\}_{\mathrm{i}=1 \ldots \mathrm{N}+1}$ are computed, the current is calculated by rearranging Eq. (20) in the following form:

$$
\begin{aligned}
J_{v}(\tau)=K_{g} \sum_{i=1}^{N} w\left(\tau, u_{i}\right) \int_{u_{i}}^{u_{1+1}} \frac{\exp \left(-u^{\prime} / \tau_{N}\right)}{\tau_{N}} 2 \pi Y\left(\tau, u^{\prime}\right)^{2} d u^{\prime}+ \\
K_{g} w\left(\tau, u_{N+1}\right) \int_{u_{N+1}}^{\tau} \frac{\exp \left(-u^{\prime} / \tau_{N}\right)}{\tau_{N}} 2 \pi Y\left(\tau, u^{\prime}\right)^{2} d u^{\prime}
\end{aligned}
$$

where, again, the continuous class radii $Y\left(\tau, u^{\prime}\right)$ are obtained from Eq.(21).

In the application of the proposed method, the number of integro-differential equations that must be solved to compute the radii $Y\left(\tau, u_{i}\right)$ and concentrations $w\left(\tau, u_{i}\right)$ (see previous section) proportionally increases with decreasing $\delta$, the number of integro-differential equations Eqs. (23)-(24) that must be solved at any $\tau$ being equal to the integer division $\tau \backslash \delta$. Therefore, the computational burden of the proposed method is largely dependent on the $\delta$ that must be imposed to enforce satisfactory agreement between the solution of Eqs. (22)-(23) and the solution of the "exact" multi-particle diffusion problem, Eqs. (15)(16).

Notice that, while the number of equations to be solved decreases with increasing $\delta$, no computational advantage can be attained by increasing $\delta$ starting from values larger than the nucleation characteristic time $\tau_{N}$. Using $\delta>>\tau_{N}$ corresponds indeed to assume that particles classes are characterized, irrespective of when they appear over the electrode, by identical class concentration, i.e., $\mathrm{w}\left(\tau, \tau_{\mathrm{i}}\right) \approx \mathrm{W}(\tau, 0)$ for any $\tau_{\mathrm{i}}$. Accordingly, a single integral equation Eq. (23) for $W(\tau, 0)$ and a single differential equation Eq. (22) for $Y(\tau, 0)$ need to be simultaneously solved at any $\tau$, and, thus, no reduction in the number of equations can be attained by further increasing $\delta$.

A simple strategy to numerically solve Eqs. (22)-(23) and compute the current, Eq. (24), is to discretize the time interval $[0, \tau]$ into subintervals of constant width $\Delta \tau \leq \delta$. Numerical schemes (e.g. the Euler method) can then be used to obtain an algebraic representation for the integrals in Eqs. (24) and the time derivatives in Eq. (23). This reduces the set of 
integro-differential equations Eqs. (23)-(24) to a set of algebraic equations, which can be iteratively solved with $\tau$ increased step by step of $\Delta \tau$ to compute $\left\{w\left(\tau, u_{i}\right), Y\left(\tau, u_{i}\right)\right\}_{i=1 \ldots N+1}$ at any $\tau$ of the introduced discretization.

\section{Results of the ACA model}

In what follows, we analyse the ability of the ACA model to describe electrodeposition dynamics. The analysis is here performed by comparing the solution of the ACA model with the "exact solution" of the multi-particle diffusion problem. The "exact solution" is derived by numerical simulation of Eqs. (15)-(16), while the ACA model is solved in its coarse-grained form, Eqs. (21)-(24). In order to numerically solve Eqs. (21)-(24), $\delta$ was decreased until relative differences in the computed current transients at two consecutives $\delta$ were lower than $5 \%$.

In Fig.2, the current transients predicted by Eqs. (21)-(24) from the ACA model are compared to the "exact solution" of the multi-particle diffusion problem (Eqs. (15)-(16)), and to the solution of the model proposed by Altimari and Pagnanelli (2016) [35]. The comparison is performed for the opposite cases of instantaneous nucleation, with $\tau_{N} \rightarrow 0$ (Fig.2a,c,e), and progressive (slow) nucleation, with $\tau_{N}=1000$ (Fig.2b,d,f). In the rows from top to bottom, $K_{g}$ ranges between 1000 and 10 . The current transient predicted under the assumption of negligible particle interaction (extended current density [32]), which is obviously only valid in the early stage of electrodeposition, is also included in Fig.2, for any $\left(\mathrm{K}_{\mathrm{g}}, \tau_{\mathrm{N}}\right)$ couple. Concerning the well-known analytic model by Scharifker and Mostany (1984) [24], even in its improved version by Mirkin and Nilov (1991) [32], we remark that it only applies to the case of diffusion-controlled growth, and might thus be implemented here only to predict the current transient at $\mathrm{K}_{\mathrm{g}}=1000$ (Fig.2, top row). These predictions are not included in Fig.2, however, because they are identical to those attained with the model proposed by Altimari and Pagnanelli (2016) [35]. For the same reason, we do not include in Fig.2 the predictions from the Bobbert et al. (1987) [36] model, which however only applies to the case of instantaneous nucleation and diffusion-controlled growth (Fig.2, top row, left plot).

\section{Figure-2}

In any of the panels of Fig.2, excellent agreement is found between the predictions of the "exact solution" of the multi-particle diffusion problem and the numerical solution of the 
ACA model. We also find that both models correctly reproduce the "extended current density" in the early stage of electrodeposition, as it must be. We further notice that the ACA model improves over the model by Altimari and Pagnanelli (2016) [35]. Indeed, under mixed kinetic-diffusion control (second and third row of Fig.2), the ACA model gives better prediction of the current transient for both the maximum current and its occurrence time $\tau_{\max }$, and for the current decay at late electrodeposition stage, i.e., for $\tau>\tau_{\max }$. We notice in this respect that we were unable to run the simulations of the multi-particle diffusion problem for $K_{g}$ values lower than $\sim 10$. For this reason, we did not include in Fig.2 a comparison between the model by Altimari and Pagnanelli (2016)[35] and the ACA model with $\mathrm{K}_{\mathrm{g}}<10$, where the discrepancy might even be larger than in the last row of Fig.2, but where both models could not be validated by a comparison with "first principles simulations".

It must be remarked that the ACA model reduces in the case of instantaneous nucleation to a couple of equations for a single radius $Y(\tau)$ and a single surface concentration $w(\tau)$ as the only unknowns to be determined (see the final comment of section 2.3). In this case, the current density is simply determined by the single particle radius $Y(\tau)$, through $J_{v}(\tau)=2 \pi /\left(3 v_{M}\right) d Y(\tau)^{3} / d \tau$. Consequently, the excellent agreement between the current predicted by the ACA model and the "exact solution" suffices, in the case of instantaneous nucleation, to ensure that the single particle radius $Y(\tau)$ coincides with the average of particle radii $\mathrm{Y}_{\mathrm{i}}(\tau)$ calculated in the multi-particle diffusion problem.

\section{Figure-3}

Unlike the case of instantaneous nucleation, the current transient is determined, in the general case of progressive nucleation (finite $\tau_{N}$ values), by all the averaged radii $Y(\tau, u)$ of the iso-nucleation-time classes $C_{u}$ (see Eq. (20)). To verify whether or not $Y(\tau, u$ ) reproduces the mean radius $\left\langle\mathrm{Y}_{\mathrm{i}}(\tau)\right\rangle_{\mathrm{u}}$ of particles nucleating over a certain small time interval around $\mathrm{u}$ in the exact multi-particle model, we report in Fig.3 both $\mathrm{Y}(\tau, \mathrm{u})$ and $\left\langle\mathrm{Y}_{\mathrm{i}}(\tau)\right\rangle_{\mathrm{u}}$. The same three cases $\mathrm{K}_{\mathrm{g}}=1000,80,10$ and $\tau_{\mathrm{N}}=1000$ as in the second column of Fig. 4 are reported in Fig.3. Once more, quite good agreement between the ACA model and the "exact solution" is found.

\section{Figure-4}

To further assess the reliability of the ACA model, in Fig. 4 we compare the quantities $\tau_{\max }$ and $\mathrm{J}_{\max }$ at various $\mathrm{K}_{\mathrm{g}}$ and $\tau_{\mathrm{N}}$ as predicted by Eqs. (17), (19) and (20) (the ACA model), 
and by the simulation of the multi-particle problem (the "exact solution"). Again, fair agreement between the predicted results is found for both the quantities $\tau_{\max }$ and $J_{\max }$ thus confirming the ability of the ACA model to properly describe electrodeposition dynamics.

\section{Conclusions}

Metal electrodeposition on foreign substrate can be modelled as a problem of diffusion to multiple hemispherical particles progressively nucleating and growing on a planar substrate. In the present work, this problem is tackled by means of the boundary integral method, and is reduced to the solution of a single integro-differential equation (combining Eqs. (17) and (19)), parameterized by the (dimensionless) particle nucleation time $u$. To obtain such equation, iso-nucleation-time classes $C_{\mathrm{u}}$ were introduced, grouping simultaneously nucleating particles, regardless of their nucleation sites; moreover, differences among the metal ion concentrations attained at particles belonging to the same iso-nucleation-time class were neglected (Averaged Class Approximation, ACA). The parameterized integro-differential equation was then reduced to a set of ordinary integrodifferential equations that could be solved numerically.

With respect to the "mean field approximation" introduced by Bobbert et al. (1987) [36], where a uniform metal ion concentration is assumed to exist at the electrode surface, our ACA model preserves (on average, at least) differences among concentrations at particles nucleating at different times, by separately tracking the evolution of iso-nucleation-time classes. It should also be emphasized that the mean field approximation by Bobbert et al. (1987) [36] is, of course, only tenable in the limit of instantaneous nucleation, i.e., with $\tau_{\mathrm{N}}$ $\rightarrow \infty$. The ACA model, on the other hand, applies for any nucleation time $\tau_{\mathrm{N}}$.

In the models proposed by Scharifker and Mostany (1984) [24] and Altimari and Pagnanelli (2016) [34,35], the analysis of the electrodeposition process is performed by the introduction of the so-called "diffusion zones" (see the Introduction section). In this approach, although the resulting mathematics is quite simple, it remains true that i) "the physical significance of these "diffusion zones" remains rather vague" (verbatim from Bobbert et al. (1987) [36]), and that ii) the growth law for any particle, for whatever nucleation time, is always imposed to be the one pertaining to an isolated particle growing under the same initial bulk concentration $c_{0}$. As a matter of fact, in the "diffusion zones" 
approach the only observable quantity is the current density, and no realistic description of the particle growth is ever claimed.

The advantages of the ACA model should now be apparent. The ACA model stems directly from a rigorous mathematical description of the physical scenario, and the approximations introduced to derive the results presented here have a clear physical meaning. Both the current density and the particle radii are obtained from the model. Information about particle size distribution and its temporal evolution is readily obtained, which might be of help to estimate nucleation rate and number density in the mixed kinetic-diffusion control regime, i.e., for $K_{g}<100$. Finally, it should be mentioned that the modest computational complexity of the numerical scheme proposed here to solve the ACA model allows for an efficient computation of the current transient generated by nucleation and growth; this constitutes a considerable advantage over the direct calculation for the multi-particle diffusion problem.

The present paper was conceived so as to give the general mathematical formulation of the ACA model, a simple numerical scheme for solving the relevant equations, and a validation of the model predictions by comparison with the direct numerical solution of the complete multi-particle simulations. A comparison of the model predictions with experimental results will be presented elsewhere. Presently, it is worth concluding by remarking that the proposed approach might offer unexplored opportunities to analyse the mechanisms of electrodeposition. The application of the ACA model or of its future developments will make it possible the introduction of complexities including, for example, the presence of species adsorbing on the surface of growing particles [39], and the nonnegligible surface diffusion of adatoms and nanoclusters [11-13].

\section{References}

[1] J.M. Petroski, Z.L. Wang, T.C. Green, M. a El-sayed, Kinetically Controlled Growth and Shape Formation Mechanism of Platinum Nanoparticles, J. Phys. Chem. B. 102 (1998) 3316-3320. doi:10.1021/jp981030f.

[2] Q. Song, Z.J. Zhang, Shape Control and Associated Magnetic Properties of Spinel Cobalt Ferrite Nanocrystals, J. Am. Chem. Soc. 126 (2004) 6164-6168. doi:10.1021/ja049931r.

[3] R. Narayanan, M.A. El-Sayed, Catalysis with transition metal nanoparticles in 
colloidal solution: Nanoparticle shape dependence and stability, J. Phys. Chem. B. 109 (2005) 12663-12676. doi:10.1021/jp051066p.

[4] P.G. Schiavi, P. Altimari, R. Zanoni, F. Pagnanelli, Morphology-controlled synthesis of cobalt nanostructures by facile electrodeposition: transition from hexagonal nanoplatelets to nanoflakes, Electrochim. Acta. 220 (2016). doi:10.1016/j.electacta.2016.10.117.

[5] U.S. Mohanty, Electrodeposition: A versatile and inexpensive tool for the synthesis of nanoparticles, nanorods, nanowires, and nanoclusters of metals, J. Appl. Electrochem. 41 (2011) 257-270. doi:10.1007/s10800-010-0234-3.

[6] M.J. Siegfried, K.S. Choi, Directing the architecture of cuprous oxide crystals during electrochemical growth, Angew. Chemie - Int. Ed. 44 (2005) 3218-3223. doi:10.1002/anie.200463018.

[7] C.H. Kuo, M.H. Huang, Morphologically controlled synthesis of Cu2O nanocrystals and their properties, Nano Today. 5 (2010) 106-116. doi:10.1016/j.nantod.2010.02.001.

[8] F. Pagnanelli, P. Altimari, M. Bellagamba, G. Granata, E. Moscardini, P.G. Schiavi, L. Toro, Pulsed electrodeposition of cobalt nanoparticles on copper: Influence of the operating parameters on size distribution and morphology, Electrochim. Acta. 155 (2015). doi:10.1016/j.electacta.2014.12.112.

[9] E. Budesky, G.T. Staikov, Electrochemical Phase Formation and Growth, VCH, Weinheim, 1996.

[10] A. Milchev, Electrocrystallization: Fundamentals of Nucleation and Growth, Kluver Academic Publishers, 2007.

[11] J. Ustarroz, J.A. Hammons, T. Altantzis, A. Hubin, S. Bals, H. Terryn, A generalized electrochemical aggregative growth mechanism, J. Am. Chem. Soc. 135 (2013) 11550-11561. doi:10.1021/ja402598k.

[12] J. Ustarroz, X. Ke, A. Hubin, S. Bals, H. Terryn, New Insights into the Early Stages of Nanoparticle Electrodeposition, J. Phys. Chem. C. 116 (2012) 2322-2329. doi:10.1021/jp210276z.

[13] S.C.S. Lai, R.A. Lazenby, P.M. Kirkman, P.R. Unwin, Nucleation, aggregative growth and detachment of metal nanoparticles during electrodeposition at electrode surfaces, Chem. Sci. 6 (2015) 1126-1138. doi:10.1039/C4SC02792B. 
[14] M.E. Hyde, R.G. Compton, A review of the analysis of multiple nucleation with diffusion controlled growth, J. Electroanal. Chem. 549 (2003) 1-12. doi:10.1016/S0022-0728(03)00250-X.

[15] M.Y. Abyaneh, Calculation of overlap for nucleation and three-dimensional growth of centres, Electrochim. Acta. 27 (1982) 1329-1334. doi:10.1016/00134686(82)80156-4.

[16] L. Guo, P.C. Searson, On the influence of the nucleation overpotential on island growth in electrodeposition, Electrochim. Acta. 55 (2010) 4086-4091. doi:10.1016/j.electacta.2010.02.038.

[17] S. Kaniyankandy, J. Nuwad, C. Thinaharan, G.K. Dey, C.G.S. Pillai, Electrodeposition of silver nanodendrites, Nanotechnology. 18 (2007). doi:10.1088/09574484/18/12/125610.

[18] W. Ye, J. Yan, Q. Ye, F. Zhou, Template-free and direct electrochemical deposition of hierarchical dendritic gold microstructures: Growth and their multiple applications, J. Phys. Chem. C. 114 (2010) 15617-15624. doi:10.1021/jp105929b.

[19] N.D. Nikolić, K.I. Popov, E.R. Ivanović, G. Branković, S.I. Stevanović, P.M. Živković, The potentiostatic current transients and the role of local diffusion fields in formation of the 2D lead dendrites from the concentrated electrolyte, J. Electroanal. Chem. 739 (2015) 137-148. doi:10.1016/j.jelechem.2014.12.020.

[20] A. Serruya, J. Mostany, B.R. Scharifker, The kinetics of mercury nucleation from $\mathrm{Hg} 22+$ and $\mathrm{Hg} 2+$ solutions on vitreous carbon electrodes, J. Electroanal. Chem. (1999). doi:10.1016/S0022-0728(98)00464-1.

[21] B.R. Scharifker, Diffusion controlled growth of hemispheres in ordered arrays, J. Electroanal. Chem. (1998). doi:10.1016/S0022-0728(98)00225-3.

[22] D. Mazaira, C. Borrás, J. Mostany, B.R. Scharifker, Three-dimensional nucleation with diffusion-controlled growth: Simulation of hierarchical diffusion zones overlap, J. Electroanal. Chem. (2009). doi:10.1016/j.jelechem.2009.03.004.

[23] B. Scharifker, G. Hills, Theoretical and experimental studies of multiple nucleation, Electrochim. Acta. 28 (1983) 879-889. doi:10.1016/0013-4686(83)85163-9.

[24] B.R. Scharifker, J. Mostany, Three-dimensional nucleation with diffusion controlled growth. Part I. Number density of active sites and nucleation rates per site, $\mathrm{J}$. Electroanal. Chem. 177 (1984) 13-23. doi:10.1016/0022-0728(84)80207-7. 
[25] J.L. Fransaer, R.M. Penner, Brownian Dynamics Simulation of the Growth of Metal Nanocrystal Ensembles on Electrode Surfaces from Solution. I. Instantaneous Nucleation and Diffusion-Controlled Growth, J. Phys. Chem. B. 103 (1999) 76437653. doi:10.1021/jp990622a.

[26] R.M. Penner, Brownian dynamics simulations of the growth of metal nanocrystal ensembles on electrode surfaces in solution: 2 . The effect of deposition rate on particle size dispersion, J. Phys. Chem. B. 105 (2001) 8672-8678.

[27] L. Guo, A. Radisic, P.C. Searson, Kinetic Monte Carlo simulations of nucleation and growth in electrodeposition., J. Phys. Chem. B. 109 (2005) 24008-15. doi:10.1021/jp055077u.

[28] Y. Cao, P.C. Searson, A.C. West, Direct Numerical Simulation of Nucleation and Three-Dimensional, Diffusion-Controlled Growth, J. Electrochem. Soc. 148 (2001) C376. doi:10.1149/1.1365144.

[29] Y. Cao, A.C. West, Nucleation and Three-Dimensional Growth: Deviation from Diffusion Control, J. Electrochem. Soc. 149 (2002) C223. doi:10.1149/1.1461379.

[30] M. Avrami, Kinetics of Phase Change. I General Theory, J. Chem. Phys. 7 (1939) 1103-1112. doi:10.1063/1.1750380.

[31] M. Sluyters-Rehbach, J.H.O.J. Wijenberg, E. Bosco, J.H. Sluyters, The theory of chronoamperometry for the investigation of electrocrystallization. Mathematical description and analysis in the case of diffusion-controlled growth, J. Electroanal. Chem. 236 (1987) 1-20. doi:10.1016/0022-0728(87)88014-2.

[32] M. V. Mirkin, A.P. Nilov, Three-dimensional nucleation and growth under controlled potential, J. Electroanal. Chem. 283 (1990) 35-51. doi:10.1016/00220728(90)87377-V.

[33] L. Heerman, A. Tarallo, Theory of the chronoamperometric transient for electrochemical nucleation with diffusion-controlled growth, J. Electroanal. Chem. 470 (1999) 70-76. doi:10.1016/S0022-0728(99)00221-1.

[34] P. Altimari, F. Pagnanelli, Electrochemical nucleation and three-dimensional growth of metal nanoparticles under mixed kinetic-diffusion control: model development and validation, Electrochim. Acta. 206 (2016). doi:10.1016/j.electacta.2016.04.094.

[35] P. Altimari, F. Pagnanelli, Electrochemical nucleation and three-dimensional growth under mixed kinetic-diffusion control: Analytical approximation of the current 
transient, Electrochim. Acta. 205 (2016). doi:10.1016/j.electacta.2016.04.093.

[36] P.A. Bobbert, M.M. Wind, J. Vlieger, Diffusion to an assembly of slowly growing particles on a substrate, Phys. A Stat. Mech. Its Appl. 146 (1987) 69-88. doi:10.1016/0378-4371(87)90223-8.

[37] A.J. Bard, L.R. Faulkner, Electrochemical Methods: Fundamentals and Applications, 2nd Ed., 2001.

[38] C.A. Brebbia, The Boundary Element Method for Engineers, London, Pe, 1980.

[39] U. Emekli, A.C. West, Simulation of the Effect of Additives on Electrochemical Nucleation, J. Electrochem. Soc. 157 (2010) D479. doi:10.1149/1.3457433.

\section{Appendix - Analytical solutions of the surface integrals in Eq. (11)}

In accordance with the assumption (see main text, assumption i), section 2.3) that metal ion concentration is uniform at the surface of any particle, the surface integrals appearing on the RHS of Eq. (11) can be rewritten as follows:

$$
\begin{aligned}
\int_{S_{i}^{\prime}} d S_{i}^{\prime} W\left(\mathbf{y}^{\prime}, \tau^{\prime}\right)\left[K_{g} \Gamma\left(\mathbf{y}, \tau \mid \mathbf{y}^{\prime}, \tau^{\prime}\right)-\mathbf{n}_{i}^{\prime} \cdot \nabla \Gamma\left(\mathbf{y}, \tau \mid \mathbf{y}^{\prime}, \tau^{\prime}\right)\right]= \\
K_{g} W_{i}\left(\tau^{\prime}\right) \int_{S_{i}^{\prime}} d S_{i}^{\prime} \Gamma\left(\mathbf{y}, \tau \mid \mathbf{y}^{\prime}, \tau^{\prime}\right)-W_{i}\left(\tau^{\prime}\right) \int_{S_{i}^{\prime}} d S_{i}^{\prime} \mathbf{n}_{i}^{\prime} \cdot \nabla \Gamma\left(\mathbf{y}, \tau \mid \mathbf{y}^{\prime}, \tau^{\prime}\right), \quad \mathbf{y} \in \bigcup_{i} S_{i}
\end{aligned}
$$

Analytical solutions for the two surface integrals on the right hand side of Eq. (A1) can be derived, which gives:

$$
\begin{gathered}
\int_{S_{i}^{\prime}} d S_{i}^{\prime} \Gamma\left(\mathbf{y}, \tau \mid \mathbf{y}^{\prime}, \tau^{\prime}\right)=\frac{Y_{i}\left[\exp \left(-\frac{\left(\left\|\mathbf{y}_{\mathbf{i}}-\mathbf{y}\right\|-Y_{i}\right)^{2}}{4\left(\tau-\tau^{\prime}\right)}\right)-\exp \left(-\frac{\left(\left\|\mathbf{y}_{\mathbf{i}}-\mathbf{y}\right\|+Y_{i}\right)^{2}}{4\left(\tau-\tau^{\prime}\right)}\right)\right]}{\sqrt{4 \pi\left(\tau-\tau^{\prime}\right)}\left\|\mathbf{y}_{\mathbf{i}}-\mathbf{y}\right\|}, \quad \mathbf{y} \in \bigcup_{i} S_{i} \\
\int_{S_{i}^{\prime}} d S_{i}^{\prime} \mathbf{n}_{\mathbf{i}}^{\prime} \cdot \nabla \Gamma\left(\mathbf{y}, \tau \mid \mathbf{y}^{\prime}, \tau^{\prime}\right)=\left[\exp \left(-\frac{\left(\left\|\mathbf{y}_{\mathbf{i}}-\mathbf{y}\right\|+Y_{i}\right)^{2}}{4\left(\tau-\tau^{\prime}\right)}\right)\left(\frac{2\left(\tau-\tau^{\prime}\right)}{\left\|\mathbf{y}_{\mathbf{i}}-\mathbf{y}\right\|}+Y_{i}+\frac{Y_{i}^{2}}{\left\|\mathbf{y}_{\mathbf{i}}-\mathbf{y}\right\|}\right)+\right. \\
\left.-\exp \left(-\frac{\left(\left\|\mathbf{y}_{\mathbf{i}}-\mathbf{y}\right\|-Y_{i}\right)^{2}}{4\left(\tau-\tau^{\prime}\right)}\right)\left(\frac{2\left(\tau-\tau^{\prime}\right)}{\left\|\mathbf{y}_{\mathbf{i}}-\mathbf{y}\right\|}-Y_{i}+\frac{Y_{i}^{2}}{\left\|\mathbf{y}_{\mathbf{i}}-\mathbf{y}\right\|}\right)\right] \frac{1}{4 \sqrt{\pi}\left(\tau-\tau^{\prime}\right)^{3 / 2}}, \quad \mathbf{y} \in \bigcup_{i} S_{i}
\end{gathered}
$$


where $\mathbf{y}_{\mathbf{i}}$ is the position occupied by the centre of particle i. Notice that $\left\|\mathbf{y}_{\mathbf{i}}-\mathbf{y}\right\|=Y_{i}$ for any $\mathbf{y} \in \mathrm{S}_{\mathrm{i}}$. In the assumption that interparticle distance is much greater than the average particle radius (see main text, assumption ii), section 2.3) it follows that $\left\|\mathbf{y}_{\mathbf{i}}-\mathbf{y}\right\| \approx\left\|\mathbf{y}_{\mathbf{i}}-\mathbf{y}_{\mathbf{j}}\right\|$ for any $\mathbf{y} \in \mathrm{S}_{\mathrm{j}}$ and $\mathrm{i} \neq \mathrm{j}$. Eqs. (A2)-(A3) can thus be approximated for any $i \neq j$ as follows:

$$
\begin{aligned}
& \int_{S_{i}^{\prime}} d S_{i}^{\prime} \Gamma\left(\mathbf{y}, \tau \mid \mathbf{y}^{\prime}, \tau^{\prime}\right) \approx \frac{Y_{i}^{2} \exp \left[-\frac{y_{i j}^{2}}{4\left(\tau-\tau^{\prime}\right)}\right]}{2 \sqrt{\pi}\left(\tau-\tau^{\prime}\right)^{3 / 2}} \\
& \int_{S_{i}^{\prime}} d S_{i}^{\prime} \mathbf{n}_{i}^{\prime} \cdot \nabla \Gamma\left(\mathbf{y}, \tau \mid \mathbf{y}^{\prime}, \tau^{\prime}\right) \approx Y_{i} \exp \left(-\frac{y_{i j}^{2}}{4\left(\tau-\tau^{\prime}\right)}\right) \frac{1}{2 \sqrt{\pi}\left(\tau-\tau^{\prime}\right)^{3 / 2}}
\end{aligned}
$$

where $y_{i j}=\left\|\mathbf{y}_{\mathbf{i}}-\mathbf{y}_{\mathbf{j}}\right\|$. Therefore, the surface integral on the left hand side of Eq. (A1) can be expressed by either Eq. (A6) or Eq. (A7) depending on whether $i \neq j$ or $i=j$, respectively, which gives:

$$
\begin{aligned}
& \begin{array}{l}
\int_{S_{i}^{\prime}} d S_{i}^{\prime} W\left(\mathbf{y}^{\prime}, \tau^{\prime}\right)\left[K_{g} \Gamma\left(\mathbf{y}, \tau \mid \mathbf{y}^{\prime}, \tau^{\prime}\right)-\mathbf{n}_{i}^{\prime} \cdot \nabla \Gamma\left(\mathbf{y}, \tau \mid \mathbf{y}^{\prime}, \tau^{\prime}\right)\right]= \\
\frac{\exp \left[-\frac{y_{i j}^{2}}{4\left(\tau-\tau^{\prime}\right)}\right]}{2 \sqrt{\pi}\left(\tau-\tau^{\prime}\right)^{3 / 2}}\left(K_{g} Y_{i}\left(\tau^{\prime}\right)^{2}-Y_{i}\left(\tau^{\prime}\right)\right) W_{i}\left(\tau^{\prime}\right), \quad i \neq j \\
\int_{S_{i}^{\prime}} d S_{i}^{\prime} W\left(\mathbf{y}^{\prime}, \tau^{\prime}\right)\left[K_{g} \Gamma\left(\mathbf{y}, \tau \mid \mathbf{y}^{\prime}, \tau^{\prime}\right)-\mathbf{n}_{i}^{\prime} \cdot \nabla \Gamma\left(\mathbf{y}, \tau \mid \mathbf{y}^{\prime}, \tau^{\prime}\right)\right]= \\
\frac{1}{\sqrt{4 \pi\left(\tau-\tau^{\prime}\right)}}\left[K_{g}+\frac{1}{Y_{i}\left(\tau^{\prime}\right)}-\left(K_{g}+\frac{1}{Y_{i}\left(\tau^{\prime}\right)}+\frac{Y_{i}\left(\tau^{\prime}\right)}{\left(\tau-\tau^{\prime}\right)}\right) \exp \left(-\frac{Y_{i}\left(\tau^{\prime}\right)^{2}}{\left(\tau-\tau^{\prime}\right)}\right)\right] W_{i}\left(\tau^{\prime}\right), i=j
\end{array}
\end{aligned}
$$

which can be substituted into Eq. (11) to obtain Eq. (15). 


\section{Figures captions}

Figure 1 - Qualitative description of the collective particle growth dynamics under diffusion and mixed kinetic-diffusion control. (A) Hemispherical particles grow independently of any other during the early stage of electrodeposition; (B) the overlapping of the hemispherical concentration fronts formed around growing particles causes the achievement of a current maximum; (C) the overlapping of hemispherical concentration fronts is completed causing the formation of a unique planar concentration front extending parallel to the electrode.

Figure 2 - Numerical validation of the ACA model. The current transient computed with the ACA model (solid line) is compared with the "exact solution" of the multi-particle diffusion problem (empty circles), the solution of the model proposed by Altimari and Pagnanelli (2016) (dashed line), and the "extended current transient" (dash-dot line). Panels on the left and on the right correspond to $\tau_{\mathrm{N}}$ equal to zero (instantaneous nucleation) and $10^{3}$, respectively; (a), (b) $K_{g}=1000$; (c), (d) $K_{g}=80$; (e), (f) $K_{g}=10$.

Figure 3 - Comparison between the particle radii predicted by the ACA model (solid lines) and by numerical solution of the multi-particle diffusion problem (empty circles). All the displayed curves were obtained with $\tau_{N}=1000$; panels (a), (b) and (c) correspond to $K_{g}$ equal to 1000,80 and 10 , respectively.

Figure 4 - Current transient characteristics $\tau_{\max }$ and $J_{\max }$ predicted by the ACA model (solid lines), by the "exact" solution of the multi-particle problem (empty squares and circles) and by the model proposed by Altimari and Pagnanelli (2016) (dash lines). Empty squares and circles correspond to $\tau_{\mathrm{N}}$ equal to 0 and 1000 , respectively. 
Figure 1

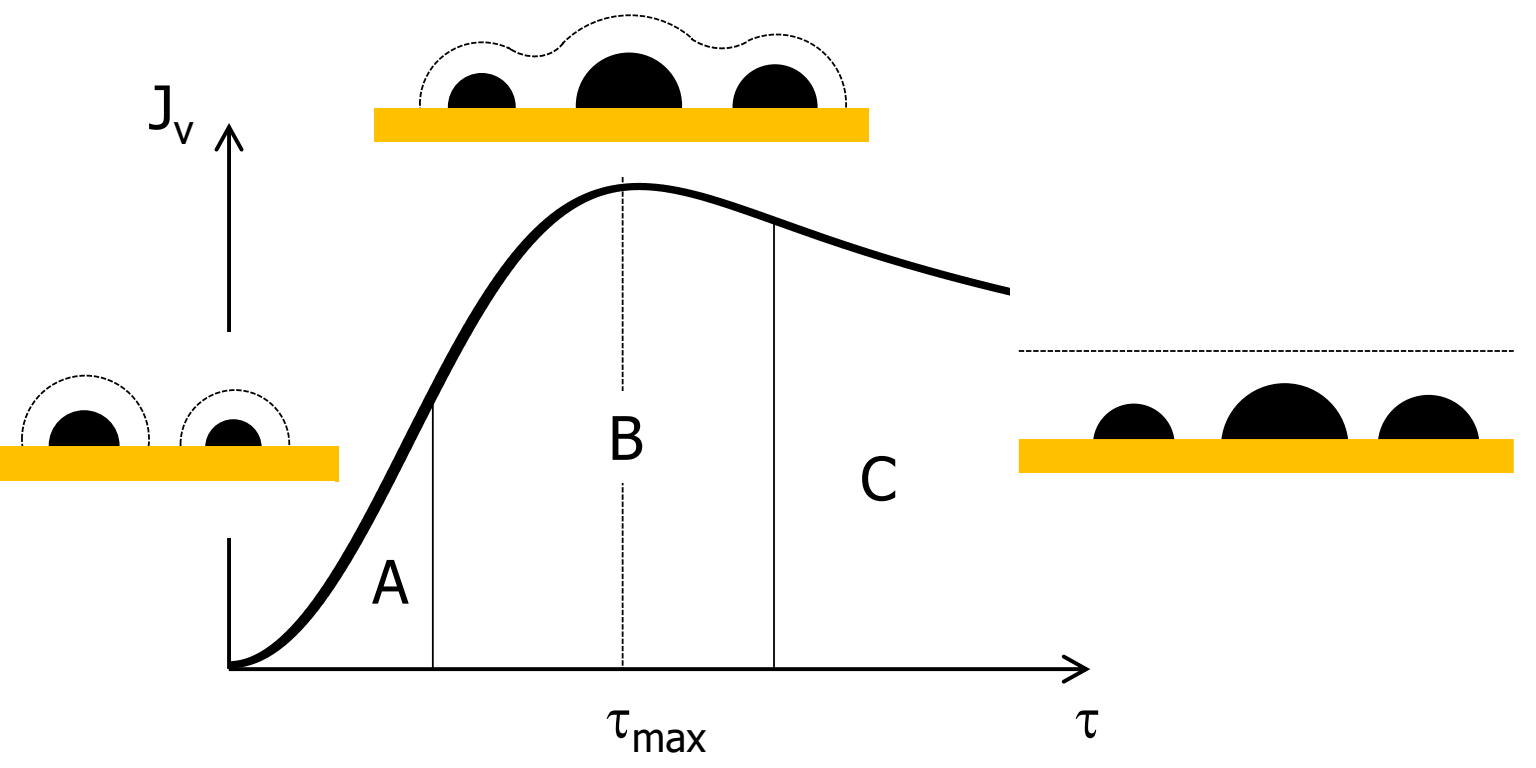


Figure 2
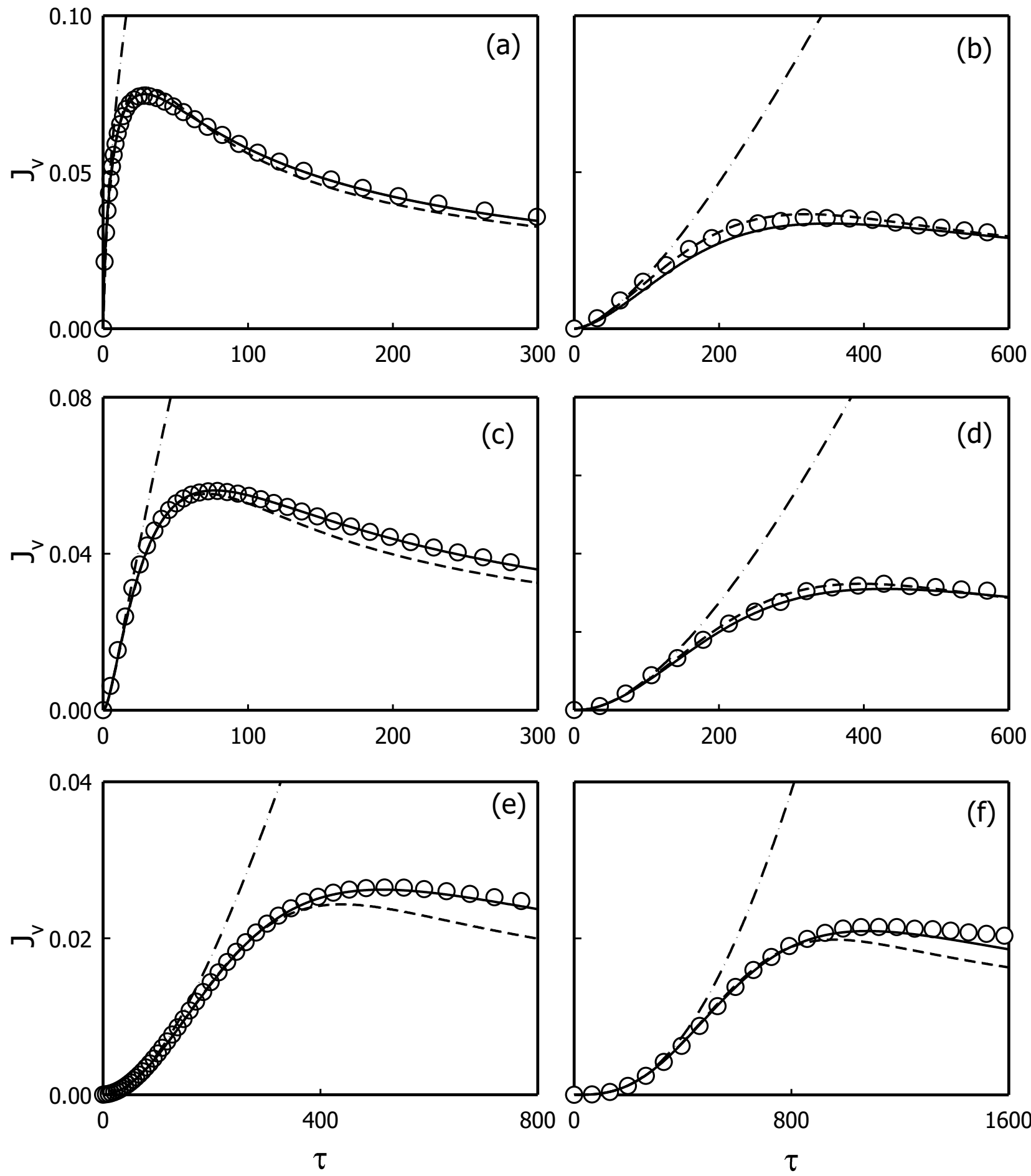
Figure 3
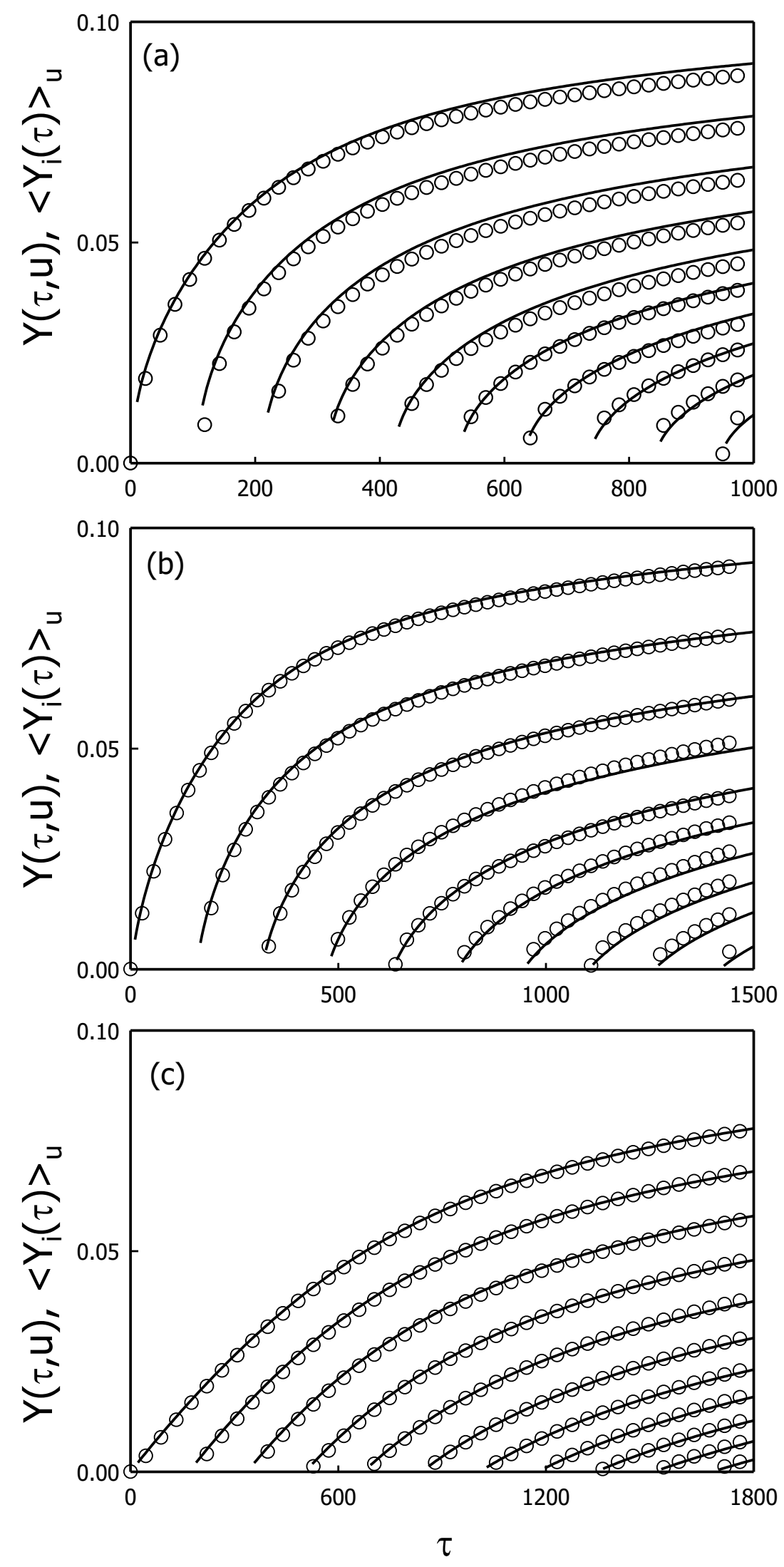
Figure 4
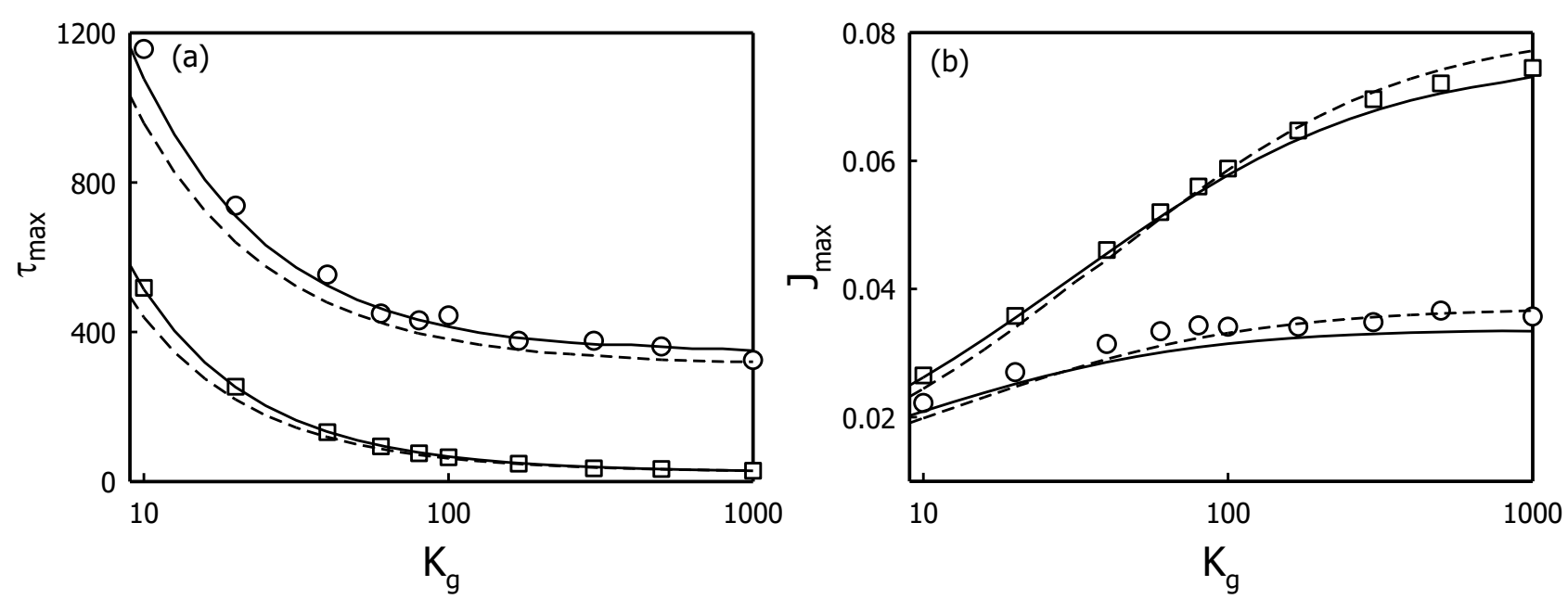